\title{
Oxidative stress is closely related to clinical severity of pre-eclampsia
}

\author{
VERÓNICA M CHAMY ${ }^{1}$, JAIME LEPE ${ }^{1}$, ÁLVARO CATALÁN ${ }^{1}$, DAVID RETAMAL ${ }^{3}$, \\ JORGE A ESCOBAR ${ }^{3}$, EVA M MADRID ${ }^{2}$
}

\footnotetext{
${ }^{1}$ Department of Gynecology and Obstetrics, School of Medicine, Universidad de Valparaíso, Chile.

${ }^{2}$ Laboratory of Morphology Sciences, School of Medicine, Universidad de Valparaíso, Chile.

${ }^{3}$ Laboratory of Biochemistry Sciences, School of Biochemistry, Pontificia Universidad Católica de Valparaiso, Chile.
}

\begin{abstract}
Pre-eclampsia is a hypertensive disorder of pregnancy in which enzymatic antioxidant defenses fail and tissues are injured. This prospective case-control study evaluated whether pre-eclamptic women and their newborns show higher degrees of oxidative stress than normal pregnancies and sought to determine if this stress is related to clinical severity. Forty-four pre-eclamptic and thirty healthy pregnant women attending two hospitals in Valparaíso, Chile, were studied.

The following plasmatic variables of antioxidant capacity were evaluated: glutathione peroxidase activity (GPx), total antioxidant capacity measured by oxidation of ABTS substrate (2,2'-azino-bis (3ethylbenzthiazoline-6-sulfonic acid), and superoxide dismutase activity (SOD). malondialdehide (MDA) was measured to evaluate lipoperoxidation. The evaluation was performed at diagnosis of pre-eclampsia, delivery, 30 days and 120 days post delivery. Newborns were studied at delivery through umbilical cord blood samples. Our analysis shows that antioxidant enzyme activity (SOD, GPx, ABTS) was significantly decreased, while lipoperoxidation (MDA) was increased in both pre-eclamptic groups compared to normal pregnant women $(\mathrm{p}<0.01)$. Statistically significant difference was found between mild and severe pre-eclamptic groups $(\mathrm{p}<0.01)$, for all biochemical markers studied. Therefore, the clinical severity of this pathology is closely related to the degree of oxidative stress.
\end{abstract}

Key terms: pre-eclampsia, MDA, SOD, GPx, ABTS, lipoperoxidation, antioxidants.

\section{INTRODUCTION}

Pre-eclampsia (PE) is a pregnancy-specific hypertension syndrome. Several studies carried out during recent years have extended our understanding about the physiopathological basis of this disease. Nevertheless, the criteria used to define it are still a matter of controversy.

Although we are able to identify the risk factors that make a pregnant woman more susceptible to develop PE, we are still unable to predict or prevent the development of the disease. In recent years, several study groups $(15,10,13)$ have published a range of guidelines and diagnostic criteria. Controversies also have arisen in relation to the criteria used to classify PE as moderate or severe $(10,13$, 19).

Oxidative stress in aerobic life can be defined as the unbalance between the generation of reactive oxygen species (ROS) and the rate of their consumption by antioxidants (8).

In normal pregnancies, there is an increase of free radical production and lipoperoxidation towards the end of pregnancy when compared with nonpregnant women. In a parallel fashion, total antioxidant capacity (Uric acid, Vitamins C and E) gradually increases during pregnancy (21), leading to an oxidative balance maintained throughout pregnancy. 
Cumulative evidence in recent years has shown that a biochemical imbalance in preeclampsia occurs with an increase of oxidative stress and lipoperoxidation and, at the same time, a deficient antioxidant protection (24).

The underlying pathogenic mechanisms of PE are the subject of many debates. The toxemia theory, which proposes that the compromised placenta produces substances leading to the maternal syndrome of PE, remains the favored hypothesis.

In normal pregnancies, there is remodeling at the placental vascular bed, where cytotrophoblast invades the maternal spiral arteries feeding intervillous space (involving endothelium and muscular tunica media), making them loose their smooth muscle and become sinusoids, a type of vessels with large capacity and low resistance, thus lacking any contractibility.

In pre-eclampsia, these physiological changes are absent. The trophoblastic invasion is very shallow, keeping the uteroplacental circulation in a state of high resistance, which results in reduced uteroplacental perfusion, focal regions of hypoxia, and alterations in the pattern of adhesion of molecules produced by endothelial cells. These changes (retention of smooth muscle caused by deficient invasion) may cause blood flow to remain under vasomotor control. The resulting larger fluctuations of intervillous oxygen concentrations might result in an ischemia-reperfusion phenomenon with overproduction of ROS (4).

In pre-eclamptic women, lipoperoxidation products (especially malondialdehyde, MDA) are increased, and enzymatic antioxidants (superoxide dismutase, SOD, and glutathione peroxidase GPx) as well as non-enzymatic antioxidants (vitamin $\mathrm{C}$ and vitamin $\mathrm{E}$ ) are decreased (22). During pre-eclampsia, levels of available nitric oxide (NO) in plasma and placenta are decreased, while an increment in nitric oxide synthase (NOS) activity occurs (12). The superoxide anion is known to inactivate $\mathrm{NO}$ in a chemical reaction forming the potent free radical peroxynitrite anion (ONOO-). The reaction of NO with superoxide is three times faster than the rate of reaction of $\mathrm{O}_{2}$ with superoxide dismutase (SOD) (22). When NO out-competes SOD for $\mathrm{O}_{2}$, production of ONOO- is favored. Peroxynitrite excess is responsible for the inflammation and nitration of tyrosine residues in proteins (nitrotyrosine) and the increase of prostaglandine synthesis as a product of the oxidation of arachidonic acid in lipidic membranes, with a subsequent blockage of vascular relaxation and increased smooth muscle contracture, leading to the hypertensive phenomena $(12,5,14)$. Generalized endothelial dysfunction may be responsible for most of the clinical manifestations of pre-eclampsia (25).

This study was designed to evaluate whether pre-eclamptic women show higher degrees of oxidative stress than normal pregnancies and to determine if this stress is related to clinical severity of preeclampsia. Finally, the study examined whether the newborns of these women showed the same biochemical alterations as their mothers.

\section{MATERIALS AND METHODS}

\section{Participants}

A case-control trial was performed in two Chilean hospitals. We recruited every preeclamptic woman admitted with pure preeclampsia (patients with no co-morbidities) in the Van Buren Hospital in Valparaíso $(n=62)$ and the Almirante Nef Naval Hospital in Viña del Mar $(n=22)$ between June 2001 and August 2003. The local ethics committee approved the study, and all women gave written informed consent, in accordance with the Helsinski criteria (14).

Thirty healthy pregnant women also were recruited to serve as the control group. Non-consenting patients, those with associated pathologies, and those lacking tests or ecographic controls were withdrawn from the protocol.

Complete study was achieved in seventy-four patients, distributed in three groups:

- Control Group (30 healthy, normal pregnant women); 
- Group with moderate PE (19 patients);

- Group of patients with severe PE (25 patients).

The following criteria for inclusion were considered:

- Control group: Healthy, normal pregnant women, non-smoking with same gestational age, similar race, age, body mass index (BMI) and weight gained during pregnancy. Health was defined as the absence of major medical or surgical disease, and no need for regular medication.

- Mild or moderate pre-eclampsia group (MPE): Blood pressure equal or higher than $140 / 90 \mathrm{mmHg}$ and under $160 / 110$ $\mathrm{mmHg}$ measured in two opportunities separated by 6 hours, urinary protein excretion over 0.3 grams/24 hours.

- Severe pre-eclampsia group (SPE): Blood pressure levels equal or over 160/ 110 , with urinary protein excretion over 0.3 grams $/ 24$ hours.

Biochemical evaluation of the mother was performed at the moment of the diagnosis (in all cases coincident with delivery, because pregnancies were interrupted upon hospital admission, 30 and 120 days post-delivery. Biochemical evaluation of the newborn was done at the moment of delivery.

Blood samples were taken by peripheric venous punction in the mother and umbilical cord blood in the newborns. Four $\mathrm{ml}$ of blood were obtained in tubes with $3 \%$ citrate as anticoagulant. The blood samples were centrifuged at $3,000 \mathrm{rpm}$ by 10 minutes, in order to separate plasma from figurate elements and placed on ice within one hour of collection.

The thiobarbituric acid assay for malondialdehyde (TBARS) (2) was performed with the plasma samples to detect oxidative damage (lipoperoxidation). Three assays were performed in plasma samples to evaluate antioxidant capacity. In the case of glutathione peroxidase, the method for assay activity GPx was used (7). Total antioxidant capacity was analyzed through the oxidation of ABTS substrate (2,2'-azino-bis(3-ethylbenzthiazoline-6sulfonic acid) (16). In the case of superoxide dismutase, the adrenochrome epinephrine detection system was used (1).

\section{Statistical analysis}

Statistical analysis between groups was determined by the non-parametric Wilcoxon signed rank test and Kruskal Wallis test. $\mathrm{P}<0.01$ was considered statistically significant.

\section{RESULTS}

\section{Quantification of lipoperoxidation (MDA)}

As shown in Figure 1-A, MDA in women with normal pregnancies show that lipoperoxidation is significantly higher compared with non-pregnant women.

At the moment of diagnosis, patients in both groups with PE showed greater MDA levels compared to normal pregnant women (Fig. 1-A). Patients with severe preeclampsia show significantly higher levels of MDA compared to moderate pre-eclampsia.

At day 30 after delivery, patients with pre-eclampsia evidence higher levels of MDA compared to normal pregnancies, and there is a significant difference between moderate and severe pre-eclampsia. Only 120 days after delivery, MDA returns to normal values in the three groups.

Newborns of patients in both groups with PE show higher levels of MDA compared to newborns of patients with normal pregnancies. Newborns of patients with severe pre-eclampsia show higher level of MDA compared to patients with moderate PE.

\section{Total antioxidant capacity (ABTS)}

As shown in Figure 2-A, women with normal pregnancies show significant higher ABTS levels compared with non-pregnant women. At diagnosis of PE, women in both groups with pre-eclampsia show significant lower ABTS than healthy pregnant women (with significant difference between severe and moderate pre-eclampsia). 

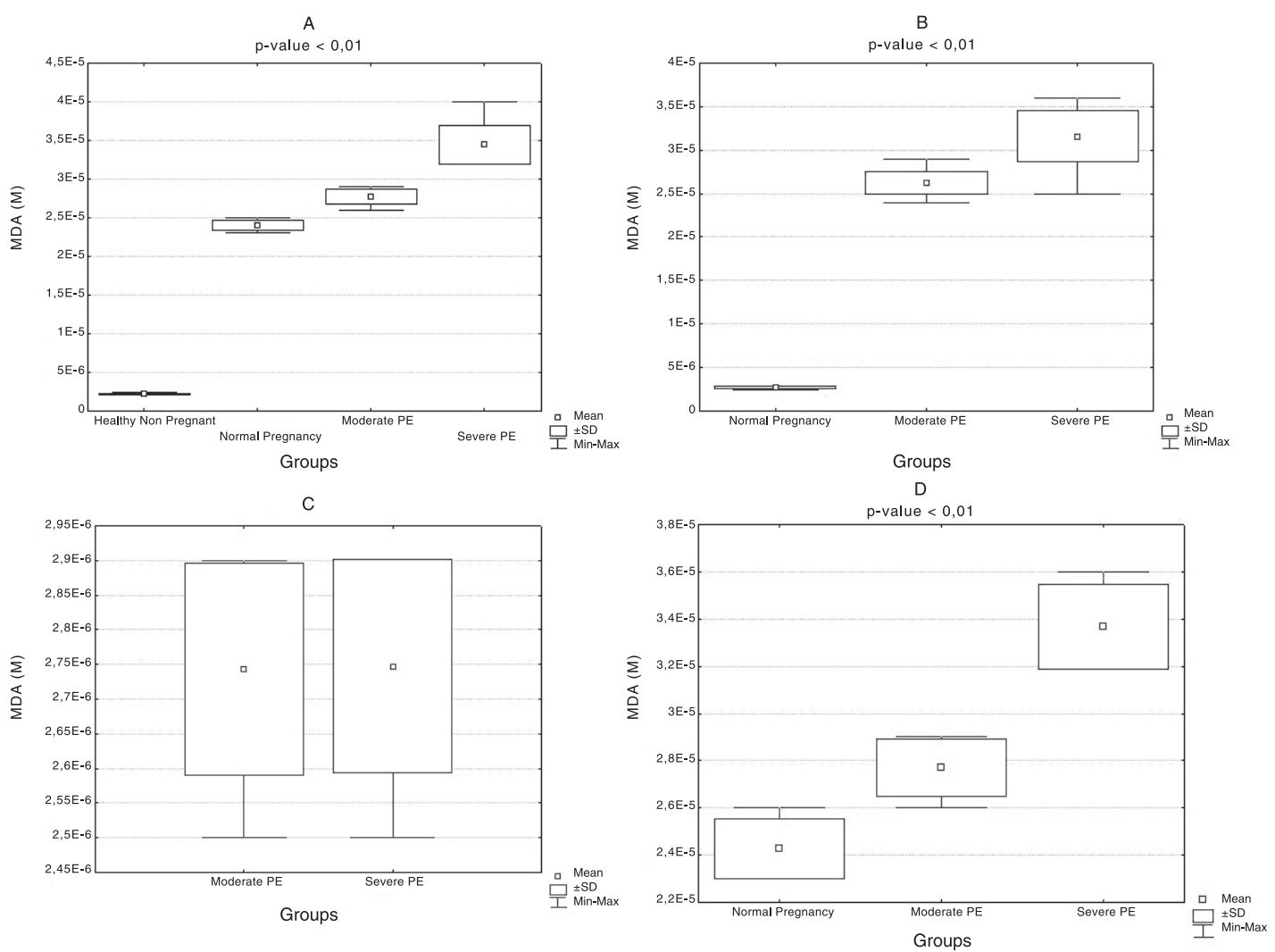

Figure 1: MDA (malon-di-aldehyde) in the three groups of pregnant women (normal pregnancies, moderate and severe preeclampsia) and newborns. Graphics show at the moment of diagnosis (A), 30 days post-delibery (B), 120 days post-delibery (C), and neeborns at delibery (D).

Thirty days after delivery, ABTS in severe and moderate pre-eclamptic women show a significant difference compared to the patients with normal pregnancies $(\mathrm{p}<0.01)$. Patients with severe preeclampsia show lower ABTS levels compared to moderate pre-eclampsia $(\mathrm{p}<0.01)$.

Four months after delivery, there was no significant difference between patients with severe and moderate pre-eclampsia. ABTS of pre-eclamptic women was within the normal range four months after delivery, while healthy pregnant women recovered pre-pregnancy levels within 30 days after delivery. We did not perform any evaluations in-between.

The newborns of both moderate and severe pre-eclamptic women show a significant difference $(\mathrm{p}<0.01)$ compared with newborns from normal pregnancies (Fig. 2-D).

\section{GPx activity (glutathione peroxidase)}

As shown in Figure 3-A, women with normal pregnancies evidence significantly higher GPx activity compared with nonpregnant women.

At the moment of diagnosis, non-pregnant women show lower degrees of GPx activity compared with healthy pregnant women. Both PE groups show lower GPx activity compared with normal pregnant women $(\mathrm{p}<0.01)$, being significantly less in the severe than in the moderate group (Fig. 3-A).

As shown in Figure 3-B, GPx activity increases 30 days after delivery in the three groups, but a significant difference continues among them. Healthy pregnant women recover pre-pregnancy levels within 30 days after delivery, while both PE groups maintain their low levels, becoming normal only after 120 days (Fig. 3-C). We did not perform any evaluation in-between. 

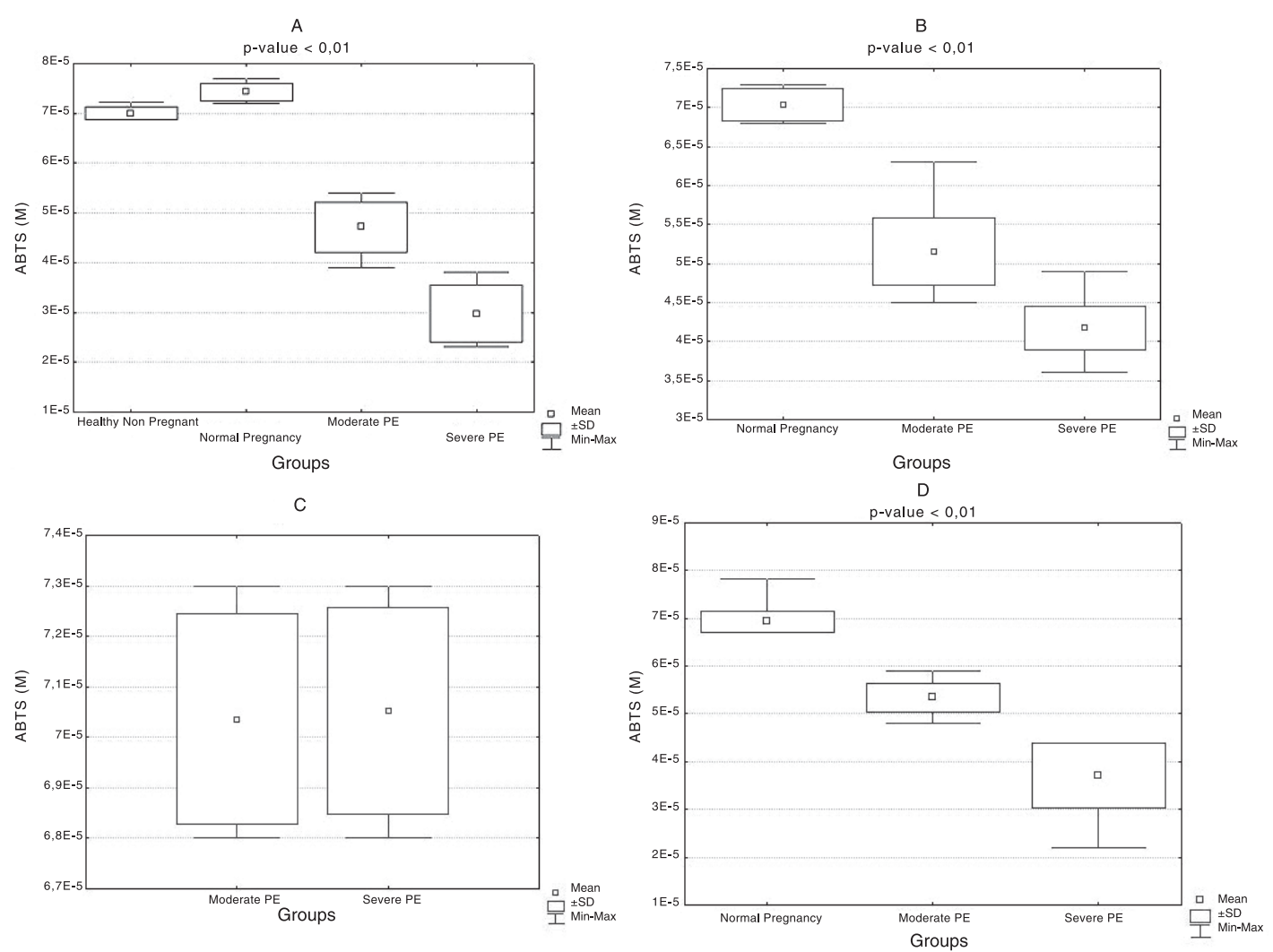

Figure 2: ABTS (Total antioxidant capacity) in the three groups of pregnant women (normal pregnancies, moderate and severe preeclampsia) and newborns. Graphics show at the moment of diagnosis (A), 30 days post-delibery (B), 120 days post-delibery (C), and neeborns at delibery (D).

In newborns, GPx activity among the three groups was statistically different $(\mathrm{p}<0.01)$, being significantly lower in both PE groups (Fig. 3-D).

\section{Superoxide dismutase activity (SOD)}

As shown in Figure 4-A, women with normal pregnancies demonstrate significantly higher SOD activity compared with non-pregnant women.

At the moment of diagnosis, patients with moderate and severe pre-eclampsia show lower SOD Activity compared with normal pregnant women (Fig. 4-A).

As shown in figure 4-B, SOD activity increases 30 days after delivery in the three groups, but a significant difference continues among them $(\mathrm{p}<0.01)$. Four months after delivery, patients with severe or moderate pre-eclampsia show no significant difference in SOD activity $(\mathrm{p}=1)$, and the values have returned to normal levels (Fig. 4-C). Healthy pregnant women recover pre-pregnancy levels within 30 days after delivery, while pre-eclamptic women only recover pre pregnancy levels at four months.

Newborns of both PE groups show a lower SOD activity compared with newborns from normal pregnancies, and the levels of SOD activity are significantly different between those from severe and moderate pre-eclamptic pregnancies (Fig. 4-D).

\section{DISCUSSION}

This case control study confirms that during normal pregnancy lipoperoxidation is increased when compared with healthy, non-pregnant women. As total antioxidant capacity (enzymatic and non-enzymatic) also increase, a compensatory balance of the injury/defense ratio is maintained. Therefore, oxidative equilibrium persists throughout pregnancy. 

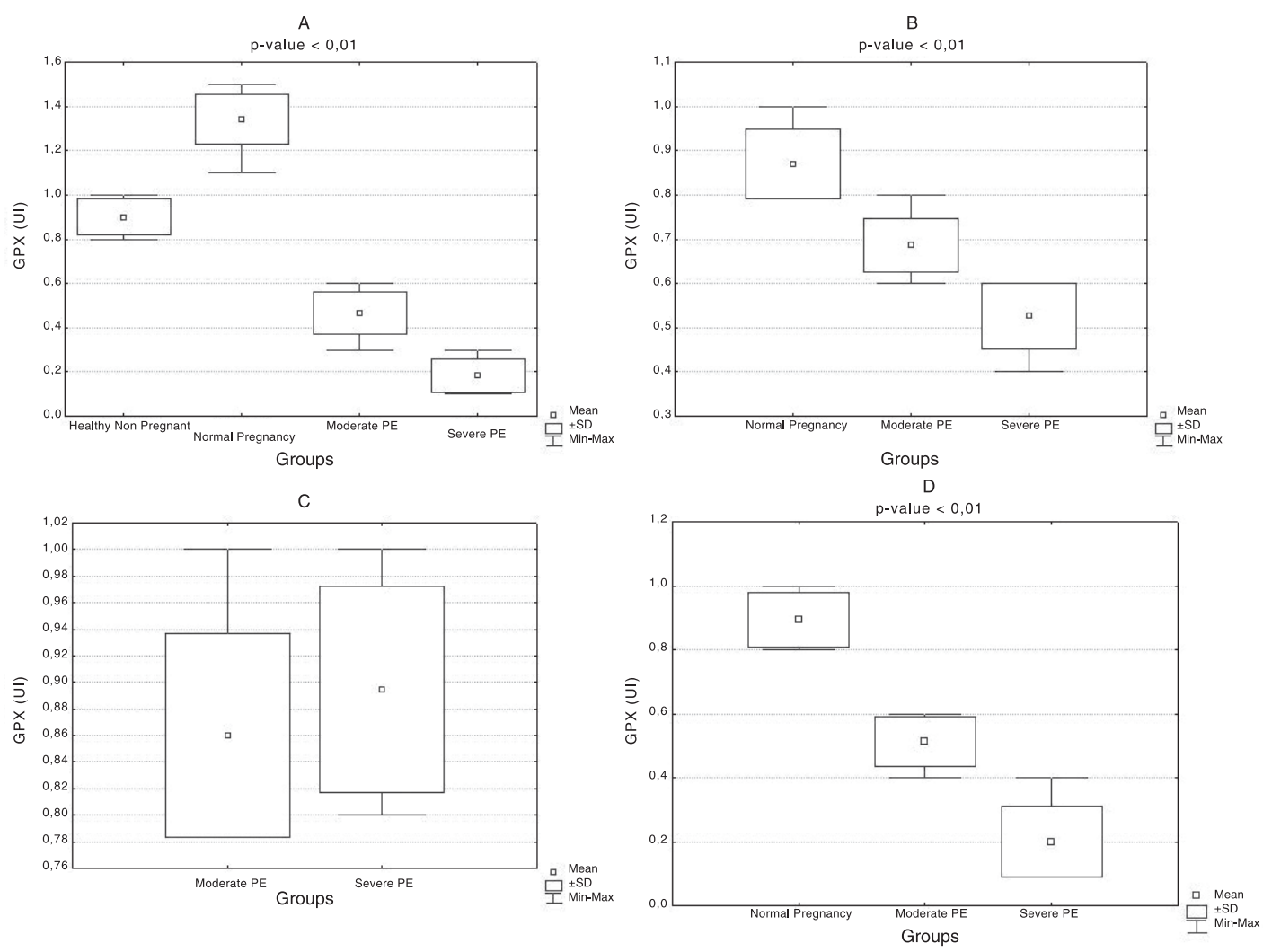

Figure 3: GPx (Ghitathione Peroxida activity) in the three groups of pregnant women (normal pregnancies, moderate and severe preeclampsia) and newborns. Graphics show at the moment of diagnosis (A), 30 days post-delibery (B), 120 days post-delibery (C), and neeborns at delibery (D).

Pre-eclamptic women fail to develop this compensatory mechanism, which was confirmed in this study by the increased MDA levels, revealing an increase in lipidic membrane damage in pre-eclamptic patients as compared with healthy pregnant patients.

This damage is more evident in the severe $\mathrm{PE}$ group compared to the mild PE group, showing a close relation between the degree of lipidic damage and clinical severity of PE. Lipidic oxidation due to PE might explain the similar morphologic changes found between PE and acute atherosis (20), and the association with future elevated cardiovascular risk, atheroma formation, and coronary arterial disease, as recurrently mentioned by many authors (11).

Recent studies showed that supplementation with antioxidants (vitamin $\mathrm{C}$ and E) may prevent endothelial dysfunction and reduce the incidence of pre-eclampsia in high-risk women (6). This study evidences the long-lasting reduction of antioxidant capacity in pre-eclamptic women, especially in severe PE. This biochemical feature does not return to normal values at day 30 after delivery, as happens in healthy pregnant women.

Although the clinical and biochemical pattern of PE disappears within one month post delivery, all biochemical oxidative stress markers do not. These indicators return to normal four months after the birth, showing that oxidative stress markers are very reliable and sensitive indexes to evidence the dramatic biochemical changes in pre-eclamptic women.

The low enzymatic SOD activity shown in the pre-eclamptic patients may be explained by the lack of induction for production of SOD, since superoxide anion is reacting with nitric oxide to produce high peroxynitrite levels (18) and, at the same time, explains the low nitric oxide levels that are usually found (12). 

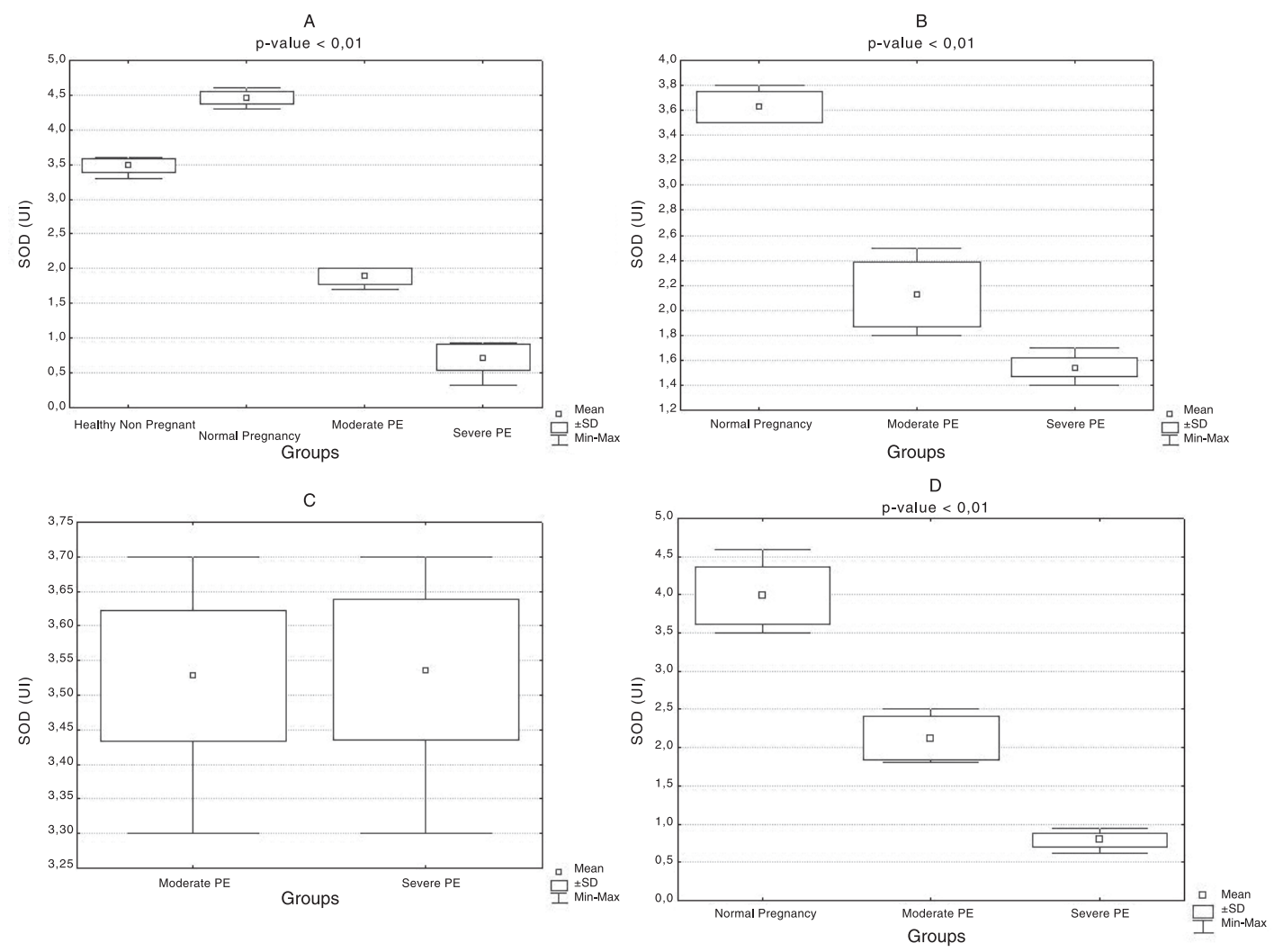

Figure 4: SOD (Superoxide Dismutase Activity) in the three groups of pregnant women (normal pregnancies, moderate and severe preeclampsia) and newborns. Graphics show at the moment of diagnosis (A), 30 days post-delibery (B), 120 days post-delibery (C), and neeborns at delibery (D).

Increasing evidence suggests that endothelial dysfunction in preeclampsia is mediated by oxidative stress $(17,23)$. Our study demonstrates a close relationship between the degree of oxidative stress and the clinical severity of this disease, since levels of lipid damage in the severe PE group was much higher than the mild PE group, suggesting that toxic effects of lipoperoxide lead to hypertension, endothelial damage, and leucocytes activation. At the same time, this research demonstrates significantly lower levels of total antioxidant capacity and enzymatic antioxidants in severe PE compared to moderate PE, confirming that the degree of biochemical disorder is closely related to clinical severity.

Barker hypothesizes that adult atherosclerosis has its origin in utero through genetic remodeling by an adverse intrauterine environment able to "program" future cardiovascular disease (3). The same significant biochemical changes observed in PE women were seen in their newborns, with differences in oxidative stress levels depending on whether they came from severe or mild PE pregnancies. These findings suggest that other factors may explain why newborns develop the same biochemical changes as their mothers, but without evidence of clinical disease. In light of Barker's hypothesis, the follow-up of these newborns in adult age would be very useful.

Pregnancy is a state of balanced increase in the production of free radicals (compensated in normal pregnancies by an increase in antioxidant defenses). Preeclampsia, in turn, represents an unbalanced state of elevated free radicals, with no antioxidant compensatory increase. Lipoperoxidation, which represents cellular lipidic damage, is significantly increased. 
All these findings are clearly and directly related to the severity of the disease.

This study supports the evidence for the role of oxidative stress in pre-eclampsia and finds substantive difference between laboratory profiles of moderate and severe PE. Future investigations are necessary to find a sensitive biochemical marker in early pregnancy of clinical usefulness as to permit interventions in order to prevent PE.

\section{ACKNOWLEDGEMENTS}

This research was performed with support of the Direction of Research (DIPUV) Universidad de Valparaíso, Chile. We gratefully acknowledge the collaboration of the Gynecology and Obstetrics Service at the Naval Hospital and Carlos Van Buren Hospital in Valparaíso, Chile and the Biochemistry Institute of the Catholic University of Valparaíso.

\section{REFERENCES}

1. ANDREWS PC, KRINSKY NI (1989) Mieloperoxidase activity. In: GREENWALD, R (ed) A CRC Hand Book by Methods for Oxygen Radical Research. 4th edition. Boca Raton, FL: CRC Press. pp: 299

2. AUST SD (1989) Lipid peroxidation. In: GREENWALD, R (ed) A CRC Hand Book by Methods for Oxygen Radical Research. 4th edition. Boca Raton, FL: CRC Press. pp: 204-206

3. Barker DJP (1995) Fetal origins of coronary heart disease. BMJ 311(15): 171-174

4. BURTON GJ, HUNG T-H (2003) Hypoxiareoxygenation: A potential source of placental oxidative stress in normal pregnancy and preeclampsia. Fetal Maternal Med Rev 14: 97-117

5. CHAMY V, MADRID E (2001) Preeclampsia y stress oxidativo: Una decáda de observaciones. Rev Chil Obstet/Ginecol 66(55): 439-444

6. CHAPPELL LC, SEED PT, BRILEY AL, KELLY FJ, LEE R, HUNT BJ, PARMAR K, BEWLEY SJ, SHENNAN AH, STEER PJ, POSTON L (1999) Effect of antioxidants on the occurrence of pre-eclampsia in women at increased risk: A randomised trial. Lancet 354: 810-816

7. DEL MAESTRO RF (1989) Oxydative enzymes in tissue homogenates. In: GREENWALD, R (ed) A CRC Hand Book by Methods for Oxygen Radical Research. 4th edition. Boca Raton, FL: CRC Press. pp: 294-296

8. FERNÁNDEZ V, VIDELA LA (1996) Biochemical aspects of cellular antioxidant systems. Biol Res 29: 177-182

9. FOOD AND DRUG ADMINISTRATION (1998) Information Sheets. The Belmont Report. Declaration of Helsinski. Update.

10. HELEWA ME, BURROWS RF, SMITH J, WILLIAMS K, BRAIN P, RABKIN SW (1997) Report of the Canadian Society Consensus Conference: 1 Definitions, evaluation and classification of hypertensive disorders in pregnancy. CMAJ 157: 71525

11. KENDALL MJ, NUTTALL SL (1999) Antioxidant therapy for the treatment of coronary artery disease. Expert opinion in investigative. Drugs 8(11): 17631784

12. LOWE DT (2000) Nitric oxide dysfunction in the pathophysiology of pre-eclampsia. Nitric Oxide 4(4): 441-8

13. AUSTRALIAN SOCIETY FOR THE STUDY OF HIGH BLOOD PRESSURE IN PREGNANCY (1993) Management of hypertension in pregnancy: Executive summary. Med J Aust 158: 700-2

14. POSTON L (2001) Oxidative stress, pre-eclampsia and antioxidants. Repro Vasc Med 1(4): 106-112

15. (2002) Report of the National High Blood Pressure Education Program Working Group on High Blood Pressure in Pregnancy. Am J Obstet Gynecol 487(1): 122

16. RICE-EVANS C, MILLER NJ (1994) Total antioxidant status in plasma and body fluids. Methods Enzymol 234: $279-293$

17. ROBERTS JM, HUBEL CA (1999) Is oxidative stress the link in the two-stage model of preeclampsia? Lancet 354: 788-789

18. ROGGENSACK AM (1999) Evidence for peroxinitrate formation in the vasculature of women with preeclampsia. Hypertension 33(1): 83-89

19. SEKIBA K, YOSHIOKA T (1977) Changes of lipid peroxidation and superoxide dismutase Activity in the human placenta. Biol Neonate 32(3-4): 147-53

20. SHANKLIN DR, SIBAI BM (1989) Ultrastructural aspects of preeclampsia: I. Placental bed and uterine boundary vessels. Am J Obstet Gynecol 161: 735-40

21. TOESCU V, NUTALL SL (2002) Oxidative Stress and Normal Pregnancy. Clinical Endocrinology 57: 609613

22. WALSH SW (1997) The role of oxidative stress and antioxidants in preeclampsia. Contemporary OB/GYN 42: $113-124$

23. WALSH SW (1998) Maternal-placenta interactions of oxidative stress and antioxidant in preeclampsia. Semin Reprod Endocrinol 16: 93-104

24. WANG Y, WALSH SW (1998) Placental mitochondria as a source of oxidative stress in pre-eclampsia. Placenta 19(8): 581-6

25. ZHOU Y, GENBACEV O, FISHER SJ (2003) The human placenta remodels the uterus by using a combination of molecules that govern vasculogenesis or leukocyte extravasation. Ann NY Acad Sci 995: 7383 